\title{
ANÁLISIS ACP, CHAID Y PLS-SEM DE LA SATISFACCIÓN DE VISITANTES DE ESPACIOS NATURALES PROTEGIDOS. APLICACIÓN AL PARQUE NACIONAL DE AIGÜESTORTES EN LLEIDA ${ }^{1}$
}

\author{
Rosa M. Florensa Guiu* \\ Universidad de Lleida \\ https://orcid.org /0000-0002-8690-0850 \\ Antonio Colom Gorgues** \\ Universidad de Lleida \\ http://orcid.org/0000-0001-7129-2539 \\ María Teresa Maza Rubio*** \\ Universidad de Zaragoza \\ https://orcid.org/0000-0002-2266-4655
}

\section{RESUMEN}

La satisfacción de los visitantes de ENP, está influenciada por variables cuantitativas y cualitativas que deben identificarse y analizarse para su optimización. El objeto de este trabajo es analizarlas en el uso público de los ENP, en aplicación al Parque Nacional de Aigüestortes.

Se han estudiado las variables explicativas con el Análisis de Componentes Principales, la Chi-cuadrado Detección Automática de Interacciones y el PLS-SEM, para valorar su influencia y explicación de la satisfacción y fidelización del visitante ecoturístico.

Fecha de recepción: 22 de diciembre de 2018.

Fecha de aceptación: 21 de junio de 2019.

* Departamento de Administración de Empresas. Facultad de Derecho, Economía y Turismo Universitat de Lleida. C/ Jaume II, 73. 25001 LLEIDA (España).E-mail: rmflorensa@aegern.udl.es

** Departamento de Administración de Empresas. Escuela Técnica Superior de Ingeniería Agraria (ETSEA) y Facultad de Derecho, Economía y Turismo (FDET). Universidad de Lleida. ETSEA: Avenida Rovira Roure, 191. 25198 LLEIDA (España). FDET: Calle Jaume II, 73. 25001 Lleida (España). E-mail: antonio_colom@hotmail.com

*** Departamento de Ciencias Agrarias y del Medio Natural. Facultad de Veterinaria. Instituto Agroalimentario de Aragón-IA2.C/ Miguel Servet 177.50013 ZARAGOZA (España).E-mail: mazama@unizar.es

1 La investigación que ha dado lugar a este trabajo ha sido impulsada por la Obra Social "la Caixa" y Fundación Triptolemos, gracias a la beca postdoctoral otorgada a la Dra. Florensa Guiu, colaborando la Universidad de Lleida y la Universidad de Zaragoza. 
Se demuestra un gran reflejo de las variables medibles valor global, recomendaciones y satisfacción a través de la gente, en la variable dependiente Satisfacción Global del Visitante.

Palabras clave: Espacios Naturales Protegidos (ENP); satisfacción de visitantes; ACP; CHAID; PLS-SEM; turismo ecológico.

PCA, Chaid and PLS-SEM Analysis on the satisfaction of visitors of Protected Natural Spaces. Ppplication to the National Park of Aigüestortes in Lleida

\begin{abstract}
The satisfaction of PNS visitors is influenced by quantitative and qualitative variables that must be identified and analyzed for optimization. The object of this work is to analyze them in the public use of the ENP, in application to the National Park of Aigüestortes.

The explanatory variables have been studied with the Principal Components Analysis, the Chi-square Automatic Interactions Detection and the PLS-SEM, to assess their influence and explanation of the satisfaction and loyalty of the ecotourist visitor.

A great reflection of the measurable variables, global value, recommendations and satisfaction through the people, is shown in the dependent variable Global Satisfaction of the Visitor.
\end{abstract}

Keywords: Protected Natural Spaces (PNS); satisfaction of visitors; PCA; CHAID; PLS-SEM; ecotourism.

\title{
1. INTRODUCCIÓN. OBJETIVOS DEL TRABAJO
}

El turismo es en España una de las actividades más importantes de la economía del país. El desarrollo y crecimiento español no se pueden entender sin tener en cuenta el papel que ha jugado el turismo, es y ha sido uno de los principales sectores generadores de crecimiento en una economía madura como la española (García et al. 2013). Junto con Francia y Estados Unidos, España es uno de los principales receptores de los movimientos turísticos internacionales. España alcanzó en 2017 un nuevo récord en la llegada de turistas internacionales al superar los 82 millones de visitantes, según el Ministerio de Energía y Turismo. Este registro supone un incremento del 8,9\% con respecto al año anterior. Este récord de visitantes se ha vinculado a un incremento del gasto hasta los 87.000 millones de euros al año, lo que supone un aumento del 12,4\% con respecto al año anterior. Cada uno de los turistas gastó 137 euros al día durante su visita a nuestro país.

Dentro de las actividades turísticas, en general, cobra cada vez más importancia y relieve socioeconómico el turismo orientado a la naturaleza, incrementando en el tiempo el uso y disfrute de los espacios naturales (Blanco y Benayas, 1994).

En la investigación que ha dado lugar al presente trabajo, se han intentado lograr los objetivos:

1. Objetivo general: Analizar las variables e inputs a considerar en los instrumentos utilizados para el seguimiento y evaluación del uso público de los Espacios Naturales 
Protegidos, en aplicación al Parque Nacional de Aigüestortes (PNA), que sirven para identificar y valorar la satisfacción y las expectativas de los visitantes, analizando también aspectos relacionados con la calidad y la actividad ecoturística.

2. Objetivos específicos:

- Valorar si existe relación entre el conocimiento, la información previa del ENP y la planificación de las visitas con el nivel de satisfacción del visitante, y por ello, tratar de evaluar si se conoce de antemano la tipología de prestaciones y servicios que se ofrecen en ese entorno.

- Relacionar si la valoración de los servicios y las infraestructuras incide en la valoración final de la visita y en la satisfacción del usuario o visitante y su fidelización.

\section{CONTEXO TEÓRICO. TURISMO DE NATURALEZA Y ECOLÓGICO, Y SATISFACCIÓN DE LOS VISITANTES}

La evolución del turismo tradicional ha ido dando paso al fenómeno del consumo masivo de naturaleza en sus formas recreativa y turística, los destinos básicos del esparcimiento en áreas naturales que son el medio rural y los espacios naturales (Blanco y Benayas, 1994).

El modelo de turismo de naturaleza (SGT, 2004) consiste en un modelo turístico individualizado y especializado, frente a los modelos de turismo de masas. En la eclosión de términos para designar estas formas alternativas de turismo, encontramos: turismo verde, turismo rural, turismo alternativo, turismo de interior, turismo ambiental, pero dos son los conceptos que se han asentado de forma clara: el ecoturismo y turismo sostenible (Donaire, 2002), aunque en alguna literatura se genera confusión entre ellos.

El ecoturismo o turismo ecológico corresponde al turismo que se realiza motivado por la naturaleza, para descubrirla, conocerla y disfrutarla, y es la modalidad turística más idónea en los espacios naturales protegidos (Gómez-Limón et al., 2010 y Pérez de las Heras, 2004).

En lo relativo a la evaluación de la satisfacción del visitante del Parque Nacional de Aigüestortes, se ha utilizado el European Customer Satisfaction Index (ECSI), siguiendo a Tenenhaus et al. (2005). Como es sabido, está basado en la metodología del National Quality Research Center de la Universidad de Michigan, y se considera un modelo muy similar al del American Customer Satisfaction Index (ACSI), con unas leves modificaciones (Tenenhaus et al., 2005).

En este modelo ECSI, se introducen siete variables interrelacionadas: Expectativas del Consumidor, Calidad Percibida, Valor Percibido, Imagen, Quejas, Satisfacción del Consumidor y Fidelización. Las cinco primeras son las variables independientes que tratarán de explicar la Satisfacción del Consumidor y su Fidelización (variables dependientes o explicadas). Se basa en teorías y enfoques bien establecidos en el comportamiento de los clientes y se recomienda aplicar a muchas industrias diferentes, para determinar un índice de satisfacción del cliente (en nuestro caso Visitante del Parque Nacional), en la industria respectiva (Ecoturismo en un Espacio Natural Protegido). ECSI, en realidad, es una adaptación del barómetro de satisfacción del cliente sueco (Fornell, 1992) y es compatible con el Índice de Satisfacción del Cliente estadounidense (ACSI). En el apartado 7 se desarrolla 
con mayor detalle este modelo ECSI, y su utilización para el análisis aplicado con un sistema de ecuaciones estructurales (SEM).

En el comienzo de cada apartado de tratamiento y análisis de los datos de la encuesta, se efectuará un resumen del contexto teórico de los modelos aplicados.

\section{ESQUEMA DE LA ESTRATEGIA METODOLÓGICA APLICADA. ENCUES- TAS, MUESTREO, APLICACIÓN Y ANÁLISIS ESTADÍSTICO}

Después de realizar la búsqueda bibliográfica correspondiente, efectuar su análisis, y completar la exploración previa con información y datos de expertos, se continuó con el diseño y aplicación de los formularios de las encuestas utilizadas en la evaluación del uso público del Parque Nacional d'Aigüestortes i Estany de Sant Maurici (PNA) en la provincia de Lleida.

Los formularios constaban de 65 preguntas que sirvieron para medir las distintas variables planteadas, y entre las cuestiones había Preguntas de Identificación, Preguntas Sustantivas, de filtro y de control. Se tuvo en cuenta las pautas definidas por Europarc, para caracterizar el perfil del visitante (Europarc, 2005).

La muestra se calculó teniendo en cuenta el número de visitantes del Parque en los diez años previos a la realización de la encuesta, valor medio situado alrededor de 350.000 visitantes, y en base a un error de muestreo e $=5,0 \%$ y un intervalo de confianza de $95,5 \%$. El tamaño de muestra necesaria era $n=400$ encuestas correctas. Ver el Cuadro 1 con la ficha técnica del muestreo.

\section{Cuadro 1 \\ FICHA TÉCNICA DEL MUESTREO}

\begin{tabular}{|l|l|}
\hline \multicolumn{1}{|c|}{ Características } & \multicolumn{1}{c|}{ Datos } \\
\hline Población objetivo & $\begin{array}{l}\text { Población infinita }(\mathrm{P}>100.000) \text {, alrededor de } \\
350.000 \text { visitantes }\end{array}$ \\
\hline Zona donde se realizó la encuesta & $\begin{array}{l}\text { Parque Nacional de Aigüestortes y Lago de San } \\
\text { Mauricio (Lleida) }\end{array}$ \\
\hline Parámetros de Muestreo & $\mathrm{p}=\mathrm{q}=0,5 ; \mathrm{z}=1,96$ \\
\hline Error de la Muestra considerado & $\mathrm{e}=0,05(5 \%)$ \\
\hline Nivel de Confianza & $95,5 \% ; \mathrm{s}=2$ \\
\hline Tamaño de la Muestra & $\begin{array}{l}\mathrm{N}=\left(\mathrm{s}^{2} \cdot \mathrm{p} \cdot \mathrm{q}\right) / \mathrm{e}^{2}=400 \text { (se realizaron finalmente } \\
452 \text { encuestas) }\end{array}$ \\
\hline Método de recogida de información y datos & Encuesta directa por entrevista personal al visitante \\
\hline Fecha del trabajo de campo & Primavera y verano de 2013 \\
\hline
\end{tabular}

Fuente: Florensa Guiu, 2015 
Se utilizaron 452 encuestas representativas de los dos sectores del PNA (sector de Aigüestortes o sede de Boí y sector Lago de San Mauricio o sede de Espot). En una primera etapa, se aplicó el programa estadístico DYANE (Diseño y Análisis de Encuestas) en su versión 4 (Santesmases, 2009) a los datos de las encuestas, para realizar el análisis estadístico multivariante de $1^{\mathrm{a}}$ generación: Análisis de Componentes Principales (ACP) y Chi-cuadrado Detección Automática de Interacciones (CHAID).

En una segunda etapa, se reconfiguraron las variables según el modelo de causalidad del Índice Europeo de Satisfacción de Clientes (European Customer Satisfaction Index o ECSI), se utilizó el sistema relacional ECSI, se diseñó el constructo interno de variables latentes y su relación con las variables medibles, aplicando el programa SmartPLS 3 en su versión 3.2.7 (Ringle et alt., 2015) para determinar los parámetros del modelo PLS-SEM multivariante de $2^{\mathrm{a}}$ generación.

Los resultados obtenidos sirvieron para estudiar, analizar y debatir relaciones entre variables, de acuerdo con los objetivos previstos, llegando a las conclusiones. Aunque en la investigación original que fundamentó una Tesis Doctoral se realizaron 9 tipos de análisis estadísticos usando el programa DYANE v4: 1. Tabulación básica; 2. Coeficiente alfa de Cronbach; 3. Análisis de Componentes Principales; 4. Tabulación cruzada de frecuencias; 5. Tabulación cruzada de valores medios; 6. Correlación lineal; 7. Análisis de Regresión múltiple; 8. Detección Automática de Interacciones; 9. Análisis de correlaciones canónicas; en este trabajo solo se presenta una breve síntesis de lo considerado más relevante y centrado en los resultados y análisis pertinentes del Análisis de Componentes Principales y de la Chi-cuadrado Detección Automática de Interacciones (se cambió desde el AID a CHAID por sus ventajas) en relación con la satisfacción del visitante del Parque Nacional. Después, se efectúa otro tratamiento con modelos de ecuaciones estructurales (PLS-SEM), y se obtienen y se analizan los resultados para tratar de explicar la variable latente Satisfacción Global del Visitante, contrastando los mismos con los anteriores. A partir de todos estos resultados, análisis, diagnósticos y contrastación se llega a las conclusiones pertinentes.

\section{CARACTERÍSTICAS DE LA ENCUESTA Y DEFINICIÓN DE LAS VARIA- BLES. UN PRIMER ANÁLISIS DESCRIPTIVO Y DEL PERFIL DEL VISI- TANTE}

El cuestionario estaba formado por 65 preguntas correspondientes a 65 variables que se presentan en el Cuadro 2. Dicho cuestionario había sido diseñado atendiendo las recomendaciones de Europarc. A través del análisis de las estadísticas básicas se identificó el perfil de las personas encuestadas que formaron la muestra y se extrajo una primera información de interés. De los 452 participantes encuestados un 55,09\% eran del sexo masculino y un $44,91 \%$ del femenino.

Las personas encuestadas iban en pareja en un 55,75\%, un 18,36\% formaban parte de grupos, el 13,50\% eran familias (parejas con hijos) y un 11,06\% iban solos. Por este motivo en la realización del cuestionario, cuando no eran personas solas se tenía en cuenta la opinión de la persona del grupo o de la pareja que mostraba mayor predisposición e interés en responder. 
El perfil de las personas encuestadas se caracteriza por la edad que en más de un $56,86 \%$ tenían entre 26 y 45 años, y un $35,52 \%$ entre 46 y 65; la residencia habitual está en Cataluña en alrededor de un 70\% (68,58\%), el 25,66\% proceden del resto de España y se cifra en un $5 \%$ la proporción de visitantes extranjeros.

Los visitantes residentes de Catalunya eran mayoritariamente de la provincia de Barcelona en una proporción del 73,87\%, siendo la zona emisora del mayor volumen de visitantes, seguía Lleida con un 13,23\% de los visitantes (cuestión de proximidad), a continuación, Tarragona con un 9,35\%, y en última posición estaban los visitantes provenientes de Girona, que suponían tan solo un 3,55\%.

Respecto el nivel de desplazamientos y el alojamiento, un $94,47 \%$ se desplazaron a la zona con vehículo particular y se alojaban en un 45,48\% en hoteles o hostales, un 19,25\% en apartamentos, un $8,63 \%$ en campings y un $5 \%$ en los refugios, y el resto se alojaba en viviendas propias o de familiares y conocidos, incluyendo aquí también la opción de visita en el dia (no alojamiento).

Para llegar al Parque, el 68,81\% de los visitantes utilizaron los taxis públicos (opción típica y mayoritaria en este parque), un $23,01 \%$ accedieron a pie y un 7,52\% en vehículo particular (modalidad reservada para casos especiales), y el resto (solo un 0,63\%) accedieron en bicicleta.

El tiempo que dedicaron a la visita fue mayoritariamente un día en un 58,19\%, un $24,34 \%$ dedicó 2 días y un 17,26\% duró su visita tres o más días. La dedicación de menos de un día fue testimonial (un $0,21 \%$ de los visitantes).

\section{Cuadro 2}

\section{LISTADO DE VARIABLES / PREGUNTAS DEL CUESTIONARIO PRESEN- TADO A LOS VISITANTES DEL PN}

\begin{tabular}{|c|c|c|c|}
\hline $\mathbf{N}^{\mathbf{o}}$ & Variable & $\begin{array}{c}\text { Denominación / Definición o contenido de la pregunta en el } \\
\text { cuestionario }\end{array}$ & Tipo variable \\
\hline 1 & SEXO & Sexo persona encuestada & Categórica \\
\hline 2 & EDAD N $^{\circ}$ & Edad persona encuestada & Numérica \\
\hline 3 & EDAD & ¿Qué Edad tiene (intervalos)? & Categórica \\
\hline 4 & RESIDENC & ¿Dónde reside habitualmente? & Mixta (escala 1 a 5$)$ \\
\hline 5 & PROVDCAT & Provincia Catalunya & Categórica \\
\hline 6 & СОМ $\square$ САТ & Comarca catalana donde reside habitualmente & Texto \\
\hline 7 & PROV ESP & Provincia española & Texto \\
\hline 8 & PAIS & País extranjero & Texto \\
\hline 9 & LLEGDCOM & ¿Como ha llegado a la comarca? & Categórica \\
\hline 10 & LLEGDPAR & ¿Como ha llegado al PN? & Categórica \\
\hline 11 & ALOJA & ¿Dónde se aloja durante la visita al PN? & Categórica \\
\hline 12 & TIEDVIAJ & Tiempo de duración del viaje & Numérica \\
\hline 13 & TIE PARC & Duración de la visita al PN & Numérica \\
\hline 14 & TIEM $1 \square 5$ & Por lo tanto, cuanto tiempo ha estado de visita al PN & Mixta (escala 1 a 5 ) \\
\hline
\end{tabular}




\begin{tabular}{|c|c|c|c|}
\hline $\mathbf{N}^{\mathbf{o}}$ & Variable & $\begin{array}{c}\text { Denominación / Definición o contenido de la pregunta en el } \\
\text { cuestionario }\end{array}$ & Tipo variable \\
\hline 15 & CONOCER & ¿Por qué motivo conoce el PN? & Categórica \\
\hline 16 & $\mathrm{CON}+\mathrm{PARC}$ & ¿Conoce más PN? & Categórica \\
\hline 17 & RED $\square \mathrm{PN}$ & $\begin{array}{l}\text { Valoración de la información que tenía sobre la existencia de } \\
\text { una red de Parques Nacionales. }\end{array}$ & Mixta (escala 1 a 5) \\
\hline 18 & NORMAS & $\begin{array}{l}\text { Información que tenía sobre la Red de Parques Nacionales y } \\
\text { sobre el parque y sus normativas }\end{array}$ & Mixta (escala 1 a 5) \\
\hline 19 & PLANIFIC & ¿Había planificado previamente la visita? & Categórica \\
\hline 20 & MOT TRAS & $\begin{array}{l}\text { ¿Considera que el motivo de la visita es trascendental y que } \\
\text { actividad pretende realizar en el PN? }\end{array}$ & Categórica \\
\hline 21 & MOT CON & $\begin{array}{l}\text { ¿Considera que el motivo de la visita es consumista y que } \\
\text { actividad pretende realizar en el PN? }\end{array}$ & Categórica \\
\hline 22 & OBJETIVO & $\begin{array}{l}\text { ¿Cuál es su valoración del objetivo conseguido sobre la } \\
\text { realización de la actividad prevista? }\end{array}$ & Mixta (escala 1 a 5$)$ \\
\hline 23 & LUGARES & ¿Qué lugares ha visitado del PN? & Categórica \\
\hline 24 & LIMP $\square$ SIT & Valoración de la limpieza del lugar visitado & Mixta (escala 1 a 5) \\
\hline 25 & LIMP $\square$ ENT & Valoración de la limpieza del entorno & Mixta (escala 1 a 5$)$ \\
\hline 26 & SEÑAL & Valoración de la señalización de los caminos & Mixta (escala 1 a 5 ) \\
\hline 27 & CAMINO & Estado de los caminos & Mixta (escala 1 a 5) \\
\hline 28 & PAISAJE & Valoración del paisaje & Mixta (escala 1 a 5 ) \\
\hline 29 & ENTORNO & Valoración de cómo ha encontrado el entorno en general & Mixta (escala 1 a 5$)$ \\
\hline 30 & IMP COIN & $\begin{array}{l}\text { Les importa el número de coincidencias con otros grupos o } \\
\text { visitantes }\end{array}$ & Categórica \\
\hline 31 & $\mathrm{~N}^{\circ} \mathrm{COINCI}$ & $\begin{array}{l}\text { Recuerda el número de personas que ha encontrado por el } \\
\text { recorrido }\end{array}$ & Numérica \\
\hline 32 & NUM MAX & $\begin{array}{l}\text { Número máximo de personas o grupos que aceptaría encontrar } \\
\text { para su gusto }\end{array}$ & Numérica \\
\hline 33 & GENT & ¿Qué le parece la cantidad de gente que ha encontrado? & Mixta (escala 1 a 5$)$ \\
\hline 34 & INFORM & ¿Ha solicitado información en algún punto del punto el PN? & Categórica \\
\hline 35 & DOND INF & ¿Dónde ha solicitado información? & Categórica \\
\hline 36 & ACCESI & Valore la accesibilidad & Mixta (escala 1 a 5$)$ \\
\hline 37 & HORARIOS & ¿Qué le han parecido los horarios? & Mixta (escala 1 a 5 ) \\
\hline 38 & ATENDPER & Valoración sobre que le ha parecido la atención personal & Mixta (escala 1 a 5 ) \\
\hline 39 & ATENDTEL & Que la ha parecido la atención telefónica & Mixta (escala 1 a 5$)$ \\
\hline 40 & LIMP $\square$ INS & Que le ha parecido la limpieza de la instalación & Mixta (escala 1 a 5$)$ \\
\hline 41 & EXPOSI & Valoración sobre que le han parecido las exposiciones & Mixta (escala 1 a 5$)$ \\
\hline 42 & AUDIOVIS & Valoración sobre que le han parecido los audiovisuales & Categórica \\
\hline 43 & PUBLICA & ¿Ha consultado publicaciones del PN? & Categórica \\
\hline 44 & QUEDPUB & ¿Qué publicaciones ha consultado? & Categórica \\
\hline 45 & OPIN PUB & Valoración sobre su opinión de las publicaciones & Mixta (escala 1 a 5$)$ \\
\hline
\end{tabular}




\begin{tabular}{|c|c|c|c|}
\hline $\mathbf{N}^{\mathbf{o}}$ & Variable & $\begin{array}{c}\text { Denominación / Definición o contenido de la pregunta en el } \\
\text { cuestionario }\end{array}$ & Tipo variable \\
\hline 46 & OBSERVA & Observaciones sobre la información & Categórica \\
\hline 47 & OPSERSUF & $\begin{array}{l}\text { ¿Considera que son suficientes los servicios/actividades del } \\
\text { PN? }\end{array}$ & Mixta (escala 1 a 5$)$ \\
\hline 48 & FAL $\square$ SEAC & ¿Ha encontrado a faltar algún servicio o actividad? & Categórica \\
\hline 49 & FAL SERV & ¿Qué servicios cree que faltan? & Texto \\
\hline 50 & FALT ACT & ¿Qué actividades ha encontrado a faltar? & Texto \\
\hline 51 & VALSERAC & ¿Qué valoración da a los servicios/actividades del Parque? & Mixta (escala 1 a 5) \\
\hline 52 & PAGARIA & ¿Le parecería adecuado pagar para poder mantener el Parque? & Mixta (escala 1 a 5$)$ \\
\hline 53 & PAGAR $\square 6$ & 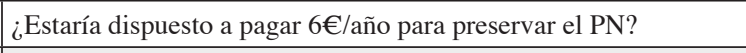 & Categórica \\
\hline 54 & $\mathrm{SI}<6 €$ & Si es menos de $6 €$ ¿¿cuánto estaría dispuesto a pagar? & Numérica \\
\hline 55 & VAL-EXPEC & ¿Se han cumplido o materializado sus expectativas sobre el PN? & Mixta (escala 1 a 5 ) \\
\hline 56 & SATGENT2 & Satisfacción en función de la gente encontrada & Mixta (escala 1 a 5$)$ \\
\hline 57 & VAL $\square$ VISI & $\begin{array}{l}\text { Valoración del nivel de satisfacción sobre los sitios visitados y su } \\
\text { valor socioeconómico }\end{array}$ & Mixta (escala 1 a 5 ) \\
\hline 58 & GUSTADO & ¿Qué le ha gustado más? & Categórica \\
\hline 59 & VALORGLO & Nivel de satisfacción total de la visita & Mixta (escala 1 a 5) \\
\hline 60 & RECOMPN & ¿Recomendaría este PN a otras personas? & Mixta (escala 1 a 5 ) \\
\hline 61 & VOLVER & Si pudiera ¿volvería a visitar el parque (u otros sitios de este)? & Mixta (escala 1 a 5$)$ \\
\hline 62 & GRUPO & Persona encuestada o grupo encuestado & Categórica \\
\hline 63 & MEJORAR & ¿Propone mejorar algún aspecto? & Categórica \\
\hline 64 & QUE MEJO & ¿Qué mejoraría? & Texto \\
\hline 65 & OBSERV & Observaciones & Texto \\
\hline
\end{tabular}

Fuente: Florensa Guiu, 2015.

Al preguntar el motivo del por qué conocían el parque, el 64,08\% indicaron que lo conocían desde siempre, un $21,29 \%$ se lo habían indicado o recomendado amigos o conocidos, y un 5,76\% les informaron en el hotel o en los puntos de información, un 4,21\% por internet y un $3,55 \%$ por revistas de montaña.

El 96\% de las personas encuestadas indicó que ya conocía otros Parques Nacionales, mencionando muchas veces el Parque Nacional de Ordesa (de relativa proximidad, ya que se encuentra en el pirineo oscense), pero solo un $66,37 \%$ expuso que conocía en mayor o menor grado la existencia de una Red de Parques Nacionales, y por otra parte, un $56.64 \%$ indicó que sabía que los Parques Nacionales tenían unos objetivos establecidos y que existían unas normas que era necesario cumplir cuando se estaba en el interior del Parque Nacional (la pregunta estaba dirigida teniendo en cuenta el momento antes de entrar en el Parque y de recibir la información en los distintos accesos).

En referencia a las actividades a realizar dentro del parque, un 72,57\% habían planificado en mayor o menor grado la visita, y un 27,43\% no tenían ninguna actividad planificada. Se diferenciaron las actividades a realizar entre trascendentales y consumistas 
(siguiendo la nomenclatura de cuestionarios utilizados anteriormente en el parque) a través de unas preguntas de respuesta múltiple y combinadas. Los que opinaron sobre actividades trascendentales, en un 50,28\% querían realizar actividades vinculadas con el senderismo y un $48,88 \%$ con el montañismo. Por otro lado, los que opinaron sobre actividades consumistas indicaron que en un $45,47 \%$ querían pasear, un $43,76 \%$ contemplar el paisaje, un $6,67 \%$ observar la flora y la fauna, y un $3,76 \%$ quería hacer fotografías.

También se preguntó sobre las posibles publicaciones que habían consultado y el $73,98 \%$ había consultado el folleto informativo gratuito que se ofrece en la entrada del parque o en los puntos de información, un $8,92 \%$ había consultado a través de Internet, un 5,58\% en Guías de Viajes, un 4,83\% en libros editados fuera del Parque y un 3,53\% en libros editados por el Parque.

Se incidió en la importancia que daban los visitantes al número de coincidencias con otros visitantes o grupos durante la vista y en los trayectos, preguntando por la cantidad máxima de coincidencias que les parecería tolerable y en contrapartida, se solicitaba su valoración para ese día concreto.

También se realizaron una serie de preguntas para saber la opinión de si la visita requería una contraprestación monetaria. Este tipo de pregunta se utilizaba hace un tiempo en la aplicación del método de la valoración contingente, que consistía en un método indirecto de valoración de los ENP en función del valor que le asignaban sus usuarios, al declarar su disposición a pagar una cantidad de dinero por esta visita, pensando en el mantenimiento y preservación del parque, y del coste que les suponía la visita en función de distintos factores.

Las preguntas sustantivas, hacían referencia a la opinión sobre los distintos aspectos relevantes relativos a las cuestiones investigadas, y recogían las valoraciones sobre las infraestructuras, los servicios, la atención, los equipamientos, el paisaje, el entorno, la limpieza, los caminos, los horarios, que guardan relación con la calidad y además las preguntas relativas con el análisis de la satisfacción y cumplimiento de las expectativas de los visitantes.

\section{ANÁLISIS DE COMPONENTES PRINCIPALES (ACP) Y SUS RESULTADOS}

El Análisis de Componentes Principales (ACP) es una técnica de análisis factorial que trata de identificar la estructura de un conjunto de variables observadas, estudiando las interrelaciones entre dichas variables con el fin de hallar un nuevo conjunto de variables, menor en número que el de las originales, que expresa las dimensiones que hay en común en las citadas variables originales. Esta técnica, permite revelar las dimensiones o factores subyacentes en la asociación o relación existente entre los valores de las variables analizadas (Santesmases, 2009; Harman, 1976; Rummel, 1970).

Por la tipología de las variables y su contenido, se priorizaron una serie de ítems a tener en cuenta, en relación a los objetivos previstos y resultó un conjunto de 16 variables, a las que se aplicó el análisis estadístico para buscar el logro de los objetivos. Estas 16 variables, que se muestran en el Cuadro 3, suponen un contenido relevante y de causalidad respecto el fin último de la explicación de la satisfacción global del visitante, y de otras variables que pueden explicar la calidad del parque y la fidelidad de este. En común, son variables mixtas medidas según una escala de Likert de 1 a 5. 


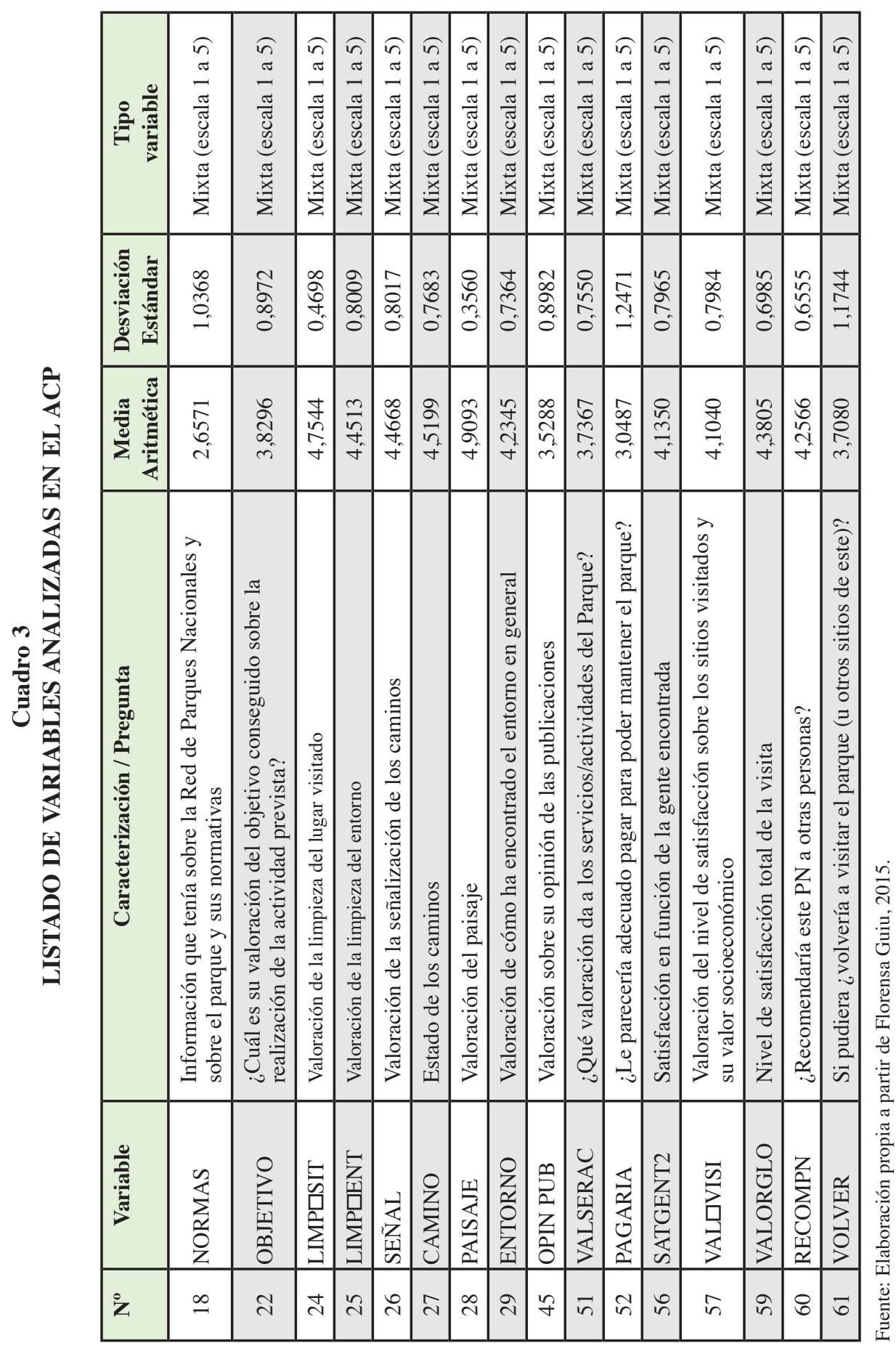


Previamente en el ACP, se calculó el valor del coeficiente de Cronbach que era 0,7027, superior a 0,7, lo cual indica una suficiente consistencia interna, y se realizó también la prueba o Test de Bartlett, a partir de la hipótesis nula para asegurar que la matriz correlación no era significativamente distinta de una matriz identidad, en la que todos los elementos son cero excepto los de la diagonal principal, que toman valor 1 .

Para la determinación de los factores, la varianza asociada con cada factor viene expresada por su valor propio, que hay tantos como variables. Como los factores no son directamente observables su denominación se basa en la carga del factor, que consiste en la correlación existente entre una variable original y un factor obtenido por combinación lineal de las variables originales. Para determinar el número de factores a retener se proponen diferentes criterios entre los que se encuentra retener los factores con raíz característica o valor propio superior a 1 (Stewart, 1981).

Posteriormente se realizó la identificación de los Valores de los factores y la Carga de los factores, aplicando la rotación de los ejes factoriales con el Método Varimax, con la caracterización de los factores resultantes e interpretación de los resultados. En el Cuadro 4 se presentan las variables y cargas de los factores retenidos, después de la rotación.

En el análisis factorial se calculó la Comunalidad para cada una de las variables originales que se obtienen sumando los cuadrados de las correlaciones o cargas de los factores retenidos con la variable para la que se calcula. Expresa la proporción de la varianza de la variable explicada con el número de factores retenidos (entre 1 y el número total de variables originales), y en total resultaron 5 factores con las 16 variables.

El primer y principal factor tenía un valor propio de 3,6135, y su explicación de la varianza de las variables es del 22,58\%. En total, se encontraron 5 Factores con valor propio superior a 1 , que explicaban en conjunto el $61,22 \%$ de la varianza.

\section{Cuadro 4}

\section{VARIABLES Y CARGAS DE LOS FACTORES RETENIDOS (DESPUÉS DE LA ROTACIÓN)}

\begin{tabular}{|c|c|c|c|c|c|c|c|}
\hline $\mathbf{N}^{\mathbf{0}}$ & VARIABLES & FAC1 & FAC2 & FAC3 & FAC4 & FAC5 & COMUNALIDAD \\
\hline 18 & NORMAS & $-0,0429$ & 0,1563 & $-0,0544$ & 0,0061 & $\mathbf{0 , 5 3 4 5} *$ & 0,3150 \\
\hline 22 & OBJETIVO & $-0,0066$ & $-0,1347$ & $0,7831^{*}$ & $-0,0164$ & $-0,0444$ & 0,6336 \\
\hline 24 & LIMP-SIT & 0,3301 & 0,0824 & $-0,0231$ & 0,2045 & $\mathbf{0 , 5 0 8 7} *$ & 0,4168 \\
\hline 25 & LIMP-ENT & $\mathbf{0 , 8 8 3 8} *$ & $-0,0312$ & 0,0306 & $-0,0868$ & 0,0075 & 0,7906 \\
\hline 26 & SENAL & $\mathbf{0 , 9 0 4 7} *$ & $-0,0378$ & 0,0298 & $-0,0953$ & 0,0051 & 0,8299 \\
\hline 27 & CAMINO & $\mathbf{0 , 7 0 4 9} *$ & 0,0768 & $-0,0325$ & 0,1019 & 0,0245 & 0,5149 \\
\hline 28 & PAISAJE & $\mathbf{0 , 5 7 7 6 *}$ & 0,1265 & 0,1117 & $-0,0459$ & $-0,2231$ & 0,4140 \\
\hline
\end{tabular}




\begin{tabular}{|c|c|c|c|c|c|c|c|}
\hline $\mathbf{N}^{\mathbf{0}}$ & VARIABLES & FAC1 & FAC2 & FAC3 & FAC4 & FAC5 & COMUNALIDAD \\
\hline 29 & ENTORNO & $\mathbf{0 , 8 0 6 4} *$ & 0,0654 & 0,0277 & $-0,0633$ & 0,1486 & 0,6814 \\
\hline 45 & OPIN PUB & 0,1863 & 0,2322 & $-0,0240$ & $\mathbf{- 0 , 7 0 5 6} *$ & $-0,2904$ & 0,6714 \\
\hline 51 & VALSERAC & 0,1226 & 0,2865 & $-0,1955$ & 0,2372 & $\mathbf{- 0 , 6 1 6 7 *}$ & 0,5719 \\
\hline 52 & PAGARIA & $-0,0001$ & 0,1943 & 0,0522 & $\mathbf{0 , 6 9 5 1 *}$ & $-0,2006$ & 0,5639 \\
\hline 56 & SATGENT2 & 0,1090 & 0,4563 & $\mathbf{0 , 7 3 1 7} *$ & 0,0550 & 0,0581 & 0,7619 \\
\hline 57 & VAL-VISI & 0,0720 & 0,4705 & $\mathbf{0 , 7 5 5 7 *}$ & 0,0846 & 0,0550 & 0,8079 \\
\hline 59 & VALORGLO & 0,1199 & $\mathbf{0 , 7 3 1 5 *}$ & 0,2312 & 0,1120 & $-0,0491$ & 0,6179 \\
\hline 60 & RECOMPN & 0,0507 & $\mathbf{0 , 7 6 0 5 *}$ & 0,0929 & 0,0005 & 0,0736 & 0,5949 \\
\hline 61 & VOLVER & $-0,0203$ & $\mathbf{0 , 7 7 7 0} *$ & $-0,0143$ & $-0,0564$ & 0,0404 & 0,6092 \\
\hline & Valor propio & 3,6135 & 2,6414 & 1,3705 & 1,1214 & 1,0483 & \\
\hline & $\%$ de varianza & $22,58 \%$ & $16,51 \%$ & $8,57 \%$ & $7,01 \%$ & $6,55 \%$ & \\
\hline & $\%$ acumulado & $22,58 \%$ & $39,09 \%$ & $47,66 \%$ & $54,67 \%$ & $61,22 \%$ & \\
\hline
\end{tabular}

Fuente: Elaboración propia a partir de Florensa Guiu, 2015.

Las asociaciones de variables que conforman los factores seleccionados son:

- El Factor 1 (F1), después de la Rotación Varimax explica al 22,58\% de la varianza, está asociado con las variables: $25,26,27,28$ y 29 . Todas estas relaciones que son positivas pueden interpretarse como la Valoración de las infraestructuras y del entorno, por lo que podría identificarse con la caracterización y evaluación de la CALIDAD PERCIBIDA.

- El Factor 2 (F2), explica el 16,51\% de la varianza, acumula el 39,09\% y está asociado con las variables 59, 60 y 61 . Todas estas relaciones que son positivas se equipararían al Producto Final o Resultado de la visita, hay una mezcla de la Satisfacción final y la Lealtad a través de las posibles recomendaciones a otras personas y la intención de volver de nuevo al espacio, por lo que podría identificarse como SATISFACCIÓN+LEALTAD $=$ RESULTADO FINAL.

- El Factor 3 (F3), explica el 8,57\% de la varianza, acumula el 47,66\%, está asociado con las variables 22,56 y 57 . Todas estas relaciones que son positivas indican la Valoración concreta de la visita en función de los objetivos definidos, por lo que podría identificarse con el VALOR PERCIBIDO

- El Factor 4 (F4), explica el 7,01\% de la varianza, acumula el 54,67\%, está asociado con las variables 45 y 52 . Presenta una correlación negativa, que indicaría un antagonismo en las opiniones que representan y que indicaría que cuando más valor económico se otorga al espacio, se tienen una opinión más crítica de las publicaciones, con lo cual se considera que esta interpretación no tiene ningún sentido, y por lo tanto se deja de lado. 
- El Factor 5 (F5), explica el 6,55\% de la varianza, acumula el 61,22\%, está asociado con las variables 18, 24 y 51. Presentan una correlación negativa con la variable 51, lo que indicaría un cierto antagonismo en las opiniones que representa la Valoración de los servicios y actividades en función del conocimiento de las normas y objetivos del espacio y que indicaría que ya conocen lo que pueden encontrarse, por lo que podría identificarse este factor con la IMAGEN.

\section{CHI-CUADRADO DETECCIÓN AUTOMÁTICA DE INTERACCIONES (CHAID) Y SUS RESULTADOS}

En la investigación original se aplicó y realizó una Detección Automática de Interacciones (AID), que corresponde a la técnica de análisis estadístico utilizada en investigación social para analizar la dependencia entre una variable criterio (variable dependiente) y múltiples predictores (variables independientes o explicativas).

En la citada investigación original, se realizaron los análisis necesarios para estudiar la relación entre la Variable Criterio que corresponde al Factor Resultado (se efectuaron tres análisis AID para cada variable criterio o dependiente: VALORGLO, RECOMPN y VOLVER) en función de las variables explicativas, diseñando los dendogramas y definiendo el perfil de los segmentos resultantes, con las Medias aritméticas y Desviaciones estándar de la variable Criterio, y cálculo del análisis de la varianza con la F de Snedecor para analizar su nivel de significación. En este trabajo, se ha optado por el análisis CHAID por sus ventajas.

El análisis Chi-cuadrado Detección Automática de Interacciones (CHAID), que es similar al análisis AID, se fundamenta en los trabajos de la Tesis Doctoral y posteriores de Kass (1980), y presenta algunas ventajas sobre esta técnica, tal como lo justifican Santesmases (2009), Magidson (1994), y Picón, Varela y Lévy (2004). Díaz-Pérez y Bethencourt-Cejas (2016) estudian y recomiendan su aplicación en el mercado turístico y su segmentación.

Además de identificar diferencias en la distribución de las respuestas de la variable criterio o dependiente sobre la base de las características de las variables predictoras o explicativas, el CHAID realiza también una partición secuencial de los elementos de la muestra, formando grupos, pero la partición no es necesariamente dicotómica como ocurre en el AID, sino que pueden formarse más de dos grupos en cada subdivisión.

Otra distinción ventajosa es que el AID se basa en un análisis de la varianza de las diferencias entre las medias de todos los grupos dicotómicos posibles. Dicho proceso de cálculo demanda determinar el estadístico F de Snedecor y elegir la partición que maximiza la varianza intergrupal; en cambio en el CHAID, al ser la variable dependiente categórica, se realizan todas las tabulaciones cruzadas posibles con las variables independientes, explicativas o predictoras, se determina en cada tabla cruzada el estadístico Chi-cuadrado y se elige la agrupación de las categorías de la variable independiente que muestra el nivel de significación más elevado.

Posteriormente reduce en uno el número de grupos pares posibles de agrupación de categorías y reteniendo la agrupación más significativa según el Chi-cuadrado y así suce- 
sivamente hasta que la agrupación en menos categorías resultante no es más significativa que la inmediatamente anterior.

\subsection{Diseño y análisis CHAID. Variables utilizadas}

Se ha definido el diseño de análisis CHAID en base a las variables criterio VALORGLO y RECOMPN, que se consideran ambas, por separado, como variables dependientes. Las variables explicativas concretas son las mismas que en el ACP, ya que suponen un contenido relevante y de causalidad respecto el fin último de la explicación de la satisfacción global del visitante y su lealtad o fidelidad (ver las variables en el Cuadro 5).

\section{Cuadro 5}

VARIABLES ANALIZADAS EN EL ANÁLISIS CHAID

\begin{tabular}{|c|c|c|c|}
\hline $\begin{array}{l}\text { Tipo de } \\
\text { variable }\end{array}$ & $\mathbf{N}^{\circ}$ & Variables & Caracterización / Pregunta \\
\hline \multirow{2}{*}{$\begin{array}{c}\text { Variables } \\
\text { criterio o } \\
\text { dependientes }\end{array}$} & 59 & VALORGLO & Nivel de satisfacción total de la visita \\
\hline & 60 & RECOMPN & ¿Recomendaría este PN a otras personas? \\
\hline \multirow{13}{*}{$\begin{array}{l}\text { Variables } \\
\text { explicativas }\end{array}$} & 18 & NORMAS & $\begin{array}{l}\text { Información que tenía sobre la Red de Parques Nacionales } \\
\text { y sobre el parque y sus normativas }\end{array}$ \\
\hline & 22 & OBJETIVO & $\begin{array}{l}\text { ¿Cuál es su valoración del objetivo conseguido sobre la } \\
\text { realización de la actividad prevista? }\end{array}$ \\
\hline & 24 & LIMP $\square$ SIT & Valoración de la limpieza del lugar visitado \\
\hline & 25 & LIMPDENT & Valoración de la limpieza del entorno \\
\hline & 26 & SEÑAL & Valoración de la señalización de los caminos \\
\hline & 27 & CAMINO & Estado de los caminos \\
\hline & 28 & PAISAJE & Valoración del paisaje \\
\hline & 29 & ENTORNO & Valoración de cómo ha encontrado el entorno en general \\
\hline & 45 & OPIN PUB & Valoración sobre su opinión de las publicaciones \\
\hline & 51 & VALSERAC & $\begin{array}{l}\text { ¿Qué valoración da a los servicios/actividades del } \\
\text { Parque? }\end{array}$ \\
\hline & 52 & PAGARIA & $\begin{array}{l}\text { ¿Le parecería adecuado pagar para poder mantener el } \\
\text { parque? }\end{array}$ \\
\hline & 56 & SATGENT2 & Satisfacción en función de la gente encontrada \\
\hline & 57 & VALDVISI & $\begin{array}{l}\text { Valoración del nivel de satisfacción sobre los sitios } \\
\text { visitados y su valor socioeconómico }\end{array}$ \\
\hline
\end{tabular}

Fuente: Elaboración propia a partir de Florensa Guiu, 2015

A continuación, se ha aplicado el análisis CHAID con el programa DYANE v4 (Santesmases, 2009), por separado una a una, respecto las dos variables criterio: VALORGLO o Nivel de Satisfacción Total de la visita, y RECOMPN o ¿Recomendaría este Parque 
Nacional a otras personas?, que indica o es sinónimo de nivel de lealtad o fidelización del visitante, y se han utilizado el mismo conjunto de variables explicativas (13 variables) que en el análisis ACP.

\subsection{Diseño y resultados CHAID VALORGLO (Nivel de Satisfacción Total de la visita) vs variables explicativas}

En el dendrograma resultado del análisis de la variable criterio VALORGLO o Nivel de Satisfacción Total de la visita, la primera variable por la que se subdivide el total de la muestra es el paisaje (PAISAJE), que es la que tiene un Chi-cuadrado más significativo en las categorías 1, 2, 4 y 5, separando la categoría 3 (paisaje de valor medio). A continuación, se agrupan 4 categorías iniciales de la variable paisaje que se muestran en el primer nivel en 3 grupos de la subdivisión que forman el segundo nivel. Estos tres grupos están formados por las 4 categorías iniciales de dicha variable Paisaje y por la variable Valoración de la visita (VAL-VISI), dividida en las categorías: 1 y 4, 2 y 3 y por último la categoría 5.

Algunas de estas agrupaciones del segundo nivel, se subdividen en un tercer nivel con la variable explicativa Valoración de la señalización de los caminos (SEÑAL), cuando las categorías de Valor de la visita son 1 y 4 (Grupo 3) y también en 5 (Grupo 5). En el grupo (Grupo 4) con las categorías 2 y 3 no se subdividen de nuevo.

El Grupo 3 se subdivide de nuevo en dos grupos, en el Grupo 6 tiene en cuenta las categorías 1, 2 ,3 y 5 y el Grupo 7 tiene en cuenta la categoría 4, pero ya no se subdividen de nuevo

El Grupo 5 se subdivide de nuevo en dos grupos, en el Grupo 8 tiene en cuenta las categorías 1, 3 ,4 y 5 y el Grupo 9 tiene en cuenta la categoría 2. El Grupo 8 tienen un valor de Chi-cuadrado elevado y se subdivide de nuevo según la variable explicativa Objetivo conseguido sobre la realización de la actividad prevista (OBJETIVO), y se ha dividido en dos grupos, el Grupo 10 tiene en cuenta las categorías 1,2 ,3 y 5 y el Grupo 11 tiene en cuenta la categoría 4.

Con lo cual, en el análisis CHAID de la Valoración Global de la visita se han agrupado las variables:

Nivel de Satisfacción Total de la visita $\rightarrow$ Paisaje $\rightarrow$ Estado de la señalización $\rightarrow$ Objetivos conseguidos 
Figura 1

REPRESENTACIÓN GRÁFICA DEL ANÁLISIS CHAID: VALORGLO / VARIABLES EXPLICATIVAS

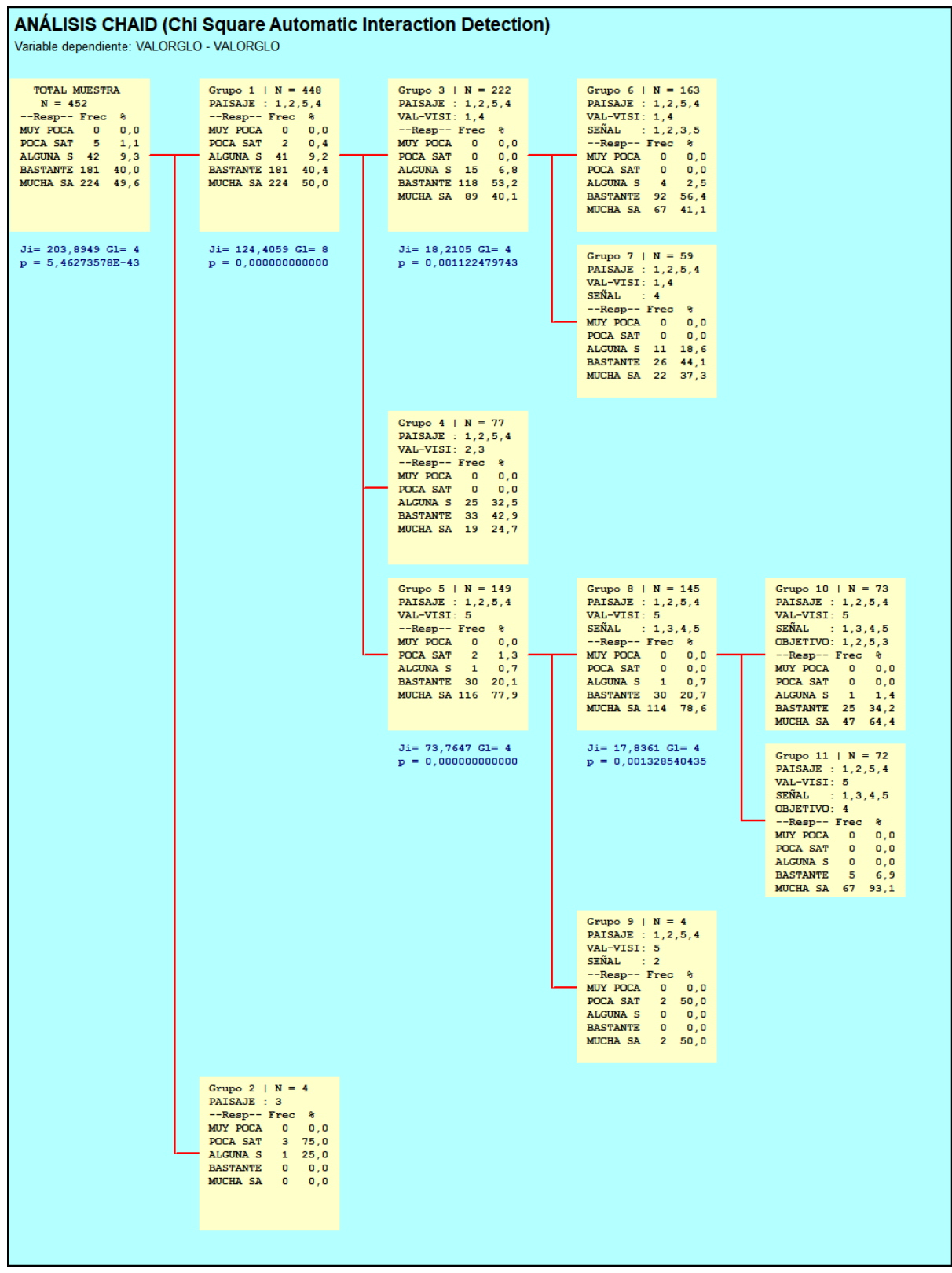

Fuente: Elaboración propia, con datos de Florensa Guiu, 2015. 
Las características de la caja del Dendrograma indican en orden secuencial las variables que han participado en la partición de los grupos, y por ejemplo en el Grupo 8, está formado por 145 individuos que valoraron el Paisaje con los criterios 1, 2, 4 y 5, valoraron la Visita con un 5 y la valoración de las Señales era 1, 3, 4 y 5. Entre estos 145 individuos, el 50\% valoró la consecución de los objetivos de la visita con 1, 2, 3 y 5, y el otro $50 \%$ con un 4.

No obstante, cabe destacar que a nivel de dendrograma se tienen en cuenta estos criterios, pero nadie valoró el Paisaje con puntuación mínima, es decir con valor igual a 1.

\subsection{Diseño y resultados CHAID RECOMPN (Recomendaría usted el Parque Nacio- nal) vs variables explicativas}

En el dendrograma resultado del análisis de la variable ¿Recomendaría usted el Parque Nacional? (RECOMPN), la primera variable por la que se subdivide el total de la muestra es la Valoración de la visita (VAL-VISI), que se divide en tres Grupos en función del Chi-cuadrado obtenido, siendo más significativo en las categorías 3, 4 (Grupo 2) y en la categoría 5 (Grupo 3), las categorías 1 y 2 están en el Grupo 1 que posteriormente no se subdivide.

A continuación, se dividen las categorías iniciales de la variable Valoración de la visita (VAL-VISI) que forman el primer nivel de la subdivisión en 2 grupos formando el segundo nivel teniendo en cuenta distintas variables. El Grupo 2 con el criterio de la Valoración de la visita 3 y 4, se subdivide según la variable Estado de los caminos (CAMINO); mientras que el Grupo 3 con el criterio 5 de la Valoración de la visita 3 se subdivide según la variable Pagaría para mantener el PN (PAGARIA).

En este segundo nivel, el Grupo 2 se subdivide en dos grupos según la variable Estado de los caminos (CAMINO), el Grupo 4 agrupa los criterios de la valoración del Estado de los caminos en 1, 2, 5 y 4, y el Grupo 5 con el criterio 3.

Por otra parte, el Grupo 3, que incluye la evaluación 5 de la Valoración de la visita, según el deseo o aceptación (parecer adecuado) de Pagar para el mantenimiento del PN (PAGARIA), se subdivide en dos grupos, el Grupo 6 agrupa los criterios de 1, 4 y 5, y el Grupo 7 con los criterios 2 y 3 . Los Grupos 4 y 5 no se subdividen, no obstante, los grupos 5 y 6 se subdividen de nuevo.

Este tercer nivel se subdivide de nuevo teniendo en cuenta distintas variables. El Grupo 6 con los criterios 1, 4 y 5 de la variable Parecer adecuado Pagar para mantener el PN (PAGARIA) se subdivide según la variable Objetivo conseguido sobre la realización de la actividad prevista (OBJETIVO) en dos grupos 8 y 9; mientras que el Grupo 7 con los criterios 2 y 3 de la variable Parecer adecuado Pagar para mantener el PN (PAGARIA) se subdivide en dos grupos: el 10 y el 11, según la variable Satisfacción en función de la gente encontrada (SATGENT2).

En el Grupo 8 se tiene en cuenta las categorías 1 y 2 de la variable Objetivo conseguido sobre la realización de la actividad prevista (OBJETIVO) y el Grupo 9 tiene en cuenta las categorías 3, 4 y 5 de la citada variable. En el Grupo 10 se tiene en cuenta las categorías 1, 2, 3 y 5 de la variable Satisfacción en función de la gente encontrada (SATGENT2) y el Grupo 11 el criterio 4 de esta. 
Figura 2

REPRESENTACIÓN GRÁFICA DEL ANÁLISIS CHAID: RECOMPN / VARIABLES EXPLICATIVAS

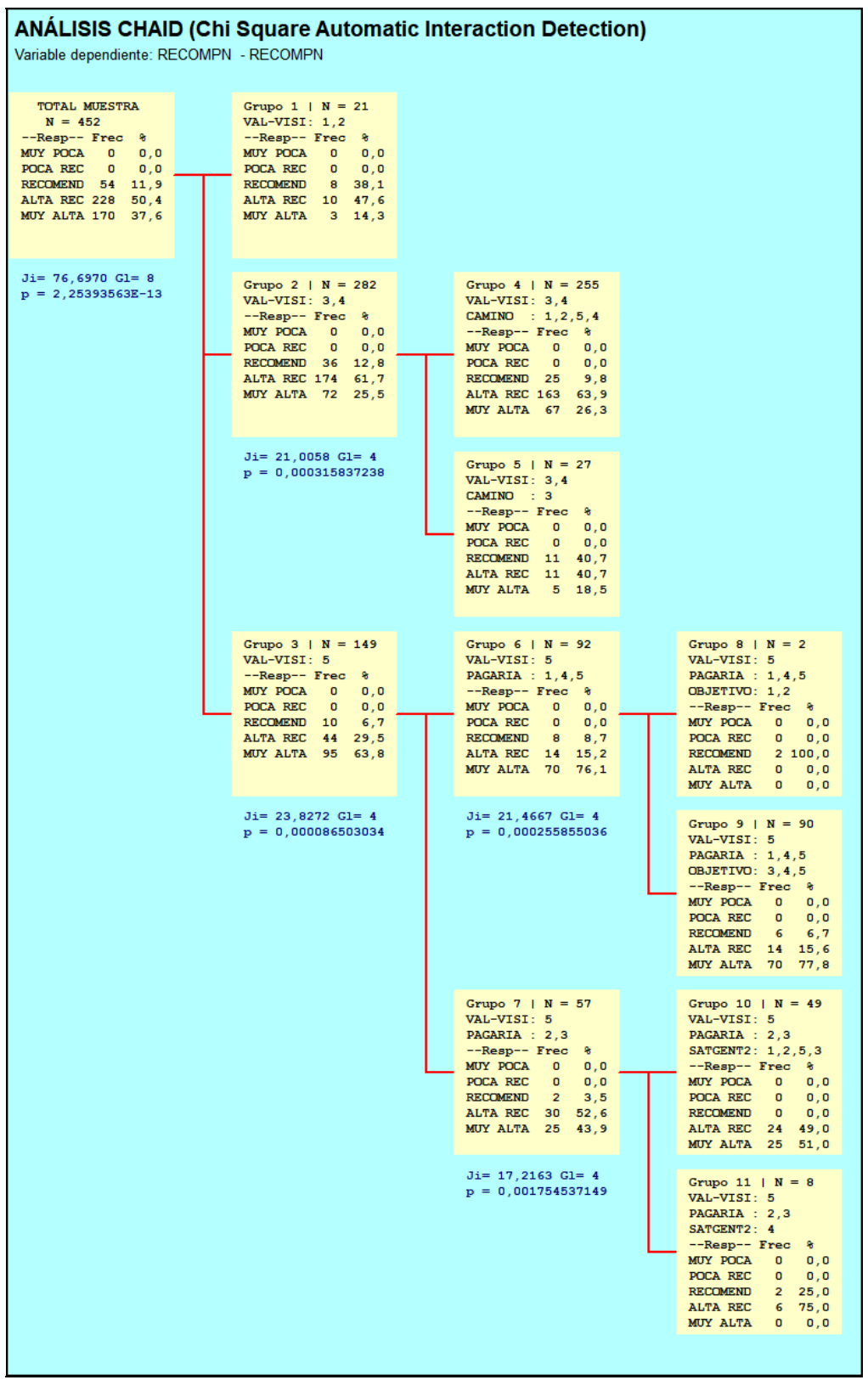

Fuente: Elaboración propia, con datos de Florensa Guiu, 2015. 
Con lo cual, en el análisis CHAID respecto la variable criterio ¿Recomendaría usted el Parque Nacional? (RECOMPN) se han agrupado las variables:

\begin{tabular}{|llll|}
\hline Recomendaría el PN $\rightarrow$ & Valoración de la visita $\rightarrow$ & $\begin{array}{l}\text { Estado camino } \\
\text { Pagaría } \rightarrow\end{array}$ & $\begin{array}{l}\text { Objetivos conseguidos } \\
\text { Satisfacción gente encontrada }\end{array}$ \\
\hline
\end{tabular}

Las características de la caja del Dendrograma indican en orden secuencial las variables que han participado en la partición de los grupos, y por ejemplo en el Grupo 6, está formado por 92 individuos que valoraron la Visita con un 5 y valoraron la posibilidad de pagar con 1, 4 y 5. Entre estos 92 individuos solo 2 valoraron la consecución de los objetivos con 1 o 2, en cambio la gran mayoría, 90, lo valoraron con 3, 4 y 5, resaltando dicho nivel de valoración.

\section{TRATAMIENTO PLS-SEM DE LA SATISFACCIÓN DEL VISITANTE. RESUL- TADO, ANÁLISIS Y DISCUSIÓN}

Se han tenido en cuenta los resultados del análisis multivariante anterior, especialmente el ACP practicado, y se ha planteado como modelo PLS-SEM, el relativo al European Customer Satisfaction Index (ECSI), siguiendo a Tenenhaus et al. (2005). Como es sabido, está basado en la metodología del National Quality Research Center de la Universidad de Michigan, y se considera un modelo muy similar al del American Customer Satisfaction Index (ACSI), al que se le han realizado algunas leves modificaciones (ver Figura 3).

\section{Figura 3}

MODELO DE CAUSALIDAD QUE DESCRIBE LAS CAUSAS Y CONSECUENCIAS DE LA SATISFACCIÓN DEL CLIENTE

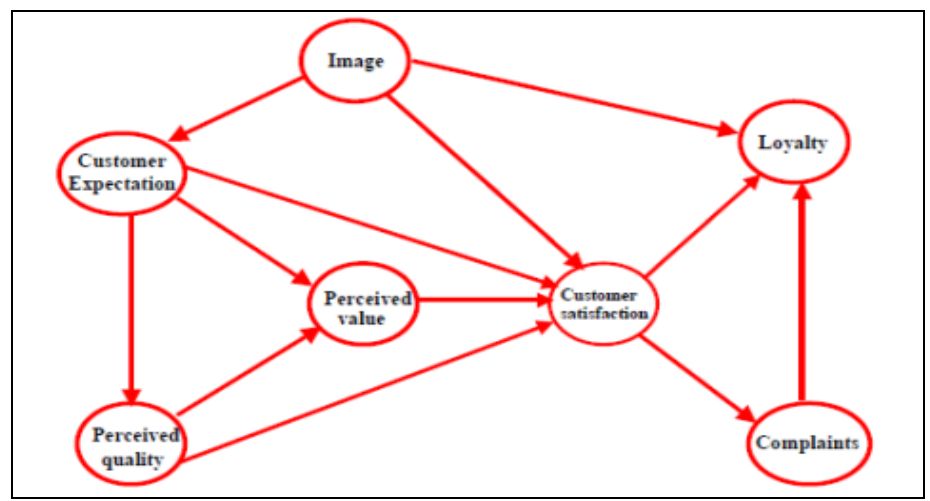

Fuente: Tenenhaus et al. (2005) (se ha conservado la nomenclatura inglesa). 
De hecho, en este modelo, se introducen siete Variables Latentes (VL) interrelacionadas, tal como se aprecia en la Figura 3. Se basa en teorías y enfoques bien establecidos en el comportamiento de los clientes y se recomienda aplicar a muchas industrias diferentes, para determinar un índice de satisfacción del cliente (en nuestro caso Visitante del Parque Nacional), en la industria respectiva (Ecoturismo en un Espacio Natural Protegido). ECSI, en realidad, es una adaptación del barómetro de satisfacción del cliente sueco (Fornell, 1992) y es compatible con el Índice de Satisfacción del Cliente estadounidense (ACSI).

Nuestro modelo PLS-SEM de partida, aplicando el ECSI anterior, se esquematiza en la Figura 4, donde se observan las siete VL interrelacionadas (constructo interno del SEM) y el conjunto de variables mesurables que permiten medir a las latentes.

\section{Figura 4}

MODELO DE PARTIDA DEL SISTEMA DE ECUACIONES ESTRUCTURALES BASADO EN EL MODELO ECSI

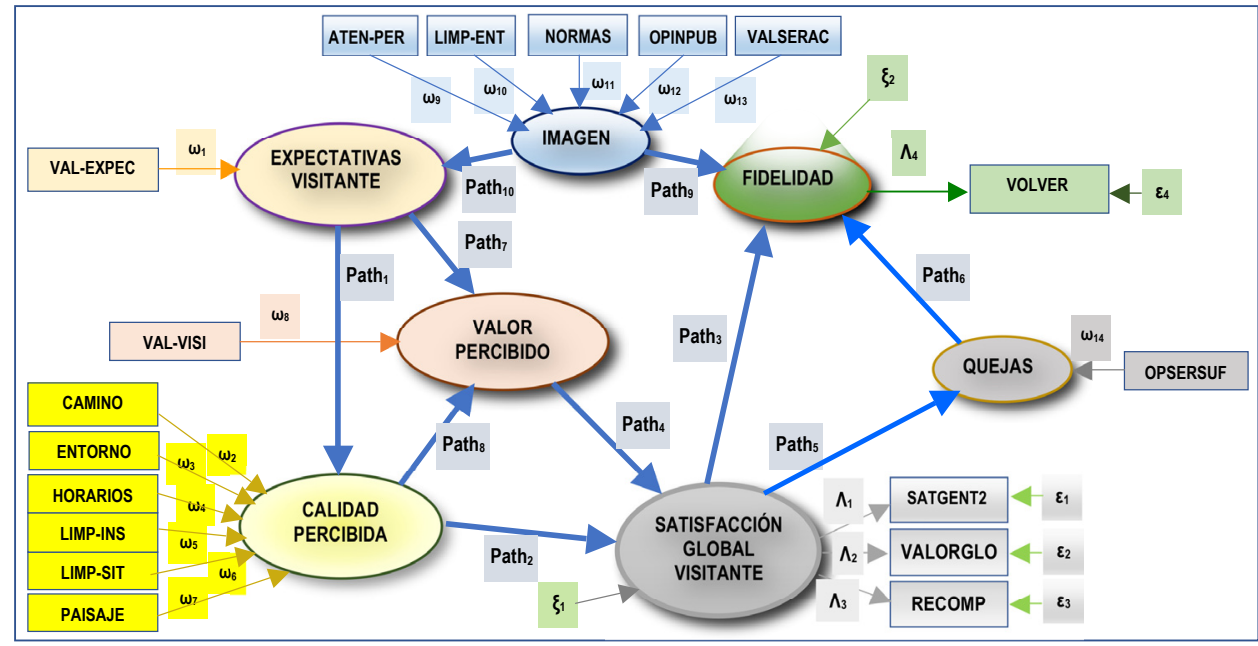

Fuente: Elaboración propia.

De acuerdo con este modelo PLS-SEM de partida que se aplica, quedan resumidas las variables latentes y las variables medibles en el Cuadro 6. En síntesis, en el constructo interno se han considerado como variables latentes dependientes la Satisfacción Global Visitante (medida por las variables mesurables VALORGLO, RECOMPN y SATGENT2) y la Fidelidad (medida por la variable mesurable VOLVER). 


\section{Cuadro 6 \\ ESPECIFICACIÓN DE VARIABLES LATENTES Y VARIABLES MEDIBLES DEL MODELO}

\begin{tabular}{|c|c|c|}
\hline $\begin{array}{l}\text { Variables } \\
\text { latentes }\end{array}$ & Var. medibles & Criterio / significación de las variables medibles \\
\hline $\begin{array}{l}\text { Expectativas } \\
\text { Visitante }\end{array}$ & VAL-EXPEC & $\begin{array}{l}\text { ¿Se han cumplido o materializado sus expectativas sobre el } \\
\text { PN? }\end{array}$ \\
\hline \multirow{6}{*}{$\begin{array}{l}\text { Calidad } \\
\text { Percibida }\end{array}$} & CAMINO & Estado de los caminos \\
\hline & ENTORNO & Valoración de cómo ha encontrado el entorno en general \\
\hline & HORARIOS & ¿Qué le han parecido los horarios? \\
\hline & LIMP-INS & Valoración de la limpieza del lugar visitado \\
\hline & LIMP-SIT & Valoración de la limpieza del entorno \\
\hline & PAISAJE & Valoración del paisaje \\
\hline $\begin{array}{l}\text { Valor } \\
\text { Percibido }\end{array}$ & VAL-VISI & $\begin{array}{l}\text { Nivel de satisfacción sobre los sitios visitados y su valor } \\
\text { socioeconómico }\end{array}$ \\
\hline \multirow{5}{*}{ Imagen } & ATEN-PER & Valoración sobre que le ha parecido la atención personal \\
\hline & LIMP-ENT & Valoración de la limpieza del entorno \\
\hline & NORMAS & $\begin{array}{l}\text { Información que tenía sobre la Red de Parques Nacionales y } \\
\text { sobre el parque y sus normativas }\end{array}$ \\
\hline & OPINPUB & Valoración sobre su opinión de las publicaciones \\
\hline & VALSERAC & ¿Qué valoración da a los servicios/actividades del Parque? \\
\hline Quejas & OPSERSUF & $\begin{array}{l}\text { ¿Opina que son suficientes los servicios y atenciones recibidos } \\
\text { del parque? }\end{array}$ \\
\hline \multirow{3}{*}{$\begin{array}{l}\text { Satisfacción } \\
\text { Global } \\
\text { Visitante }\end{array}$} & SATGENT2 & Satisfacción en función de la gente encontrada \\
\hline & VALORGLO & Nivel de satisfacción total de la visita \\
\hline & RECOMPN & ¿Recomendaría este PN a otras personas? \\
\hline Fidelidad & VOLVER & Si pudiera ¿volvería a visitar el parque (u otros sitios de este)? \\
\hline
\end{tabular}

Fuente: Elaboración propia con base en Florensa Guiu, 2015.

En lo que concierne a las variables latentes explicativas o independientes, se han considerado siguiendo el modelo ECSI: las Expectativas del Visitante (medida por la variable mesurable VAL-EXPEC); la Calidad Percibida (medida por las variables mesurables CAMINO, ENTORNO, HORARIOS, LIMP-INS, LIMP-SIT y PAISAJE); Valor Percibido (medida por la variable mesurable VAL-VISI); la Imagen (medida por el grupo de variables mesurables ATEN-PER, LIMP-ENT, NORMAS, OPINPUB y VALSERAC); y la variable Quejas (medible por la variable mesurable OPSERSUF). Todas las variables medibles eran de tipo mixto, presentando la opción de respuesta según escala de Likert de 1 a 5 . 


\section{Figura 4 \\ MODELO DE CÁLCULO Y RESULTADOS OBTENIDOS EN EL ANÁLISIS PLS-SEM CON EL PROGRAMA SmartPLS 3.2.7}

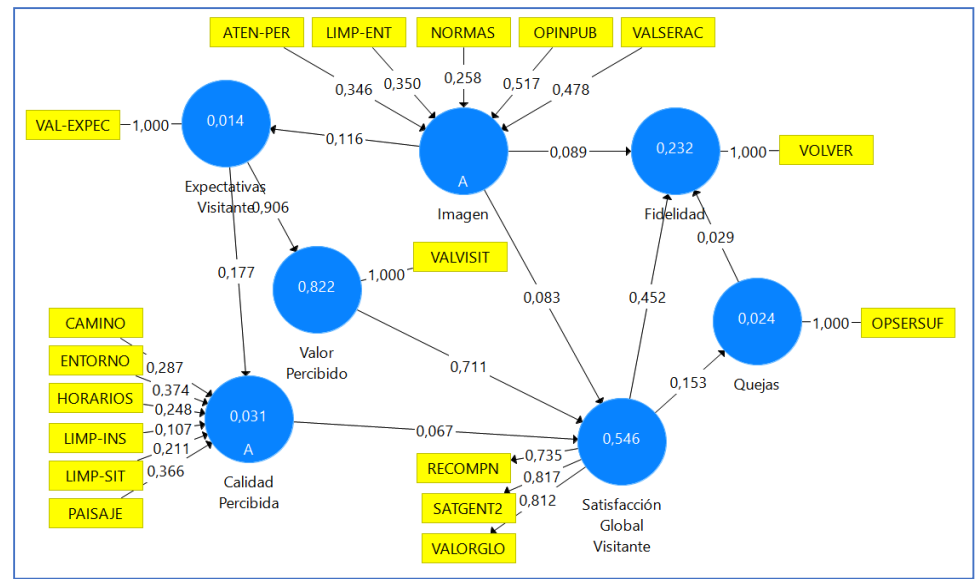

\begin{tabular}{|c|c|}
\hline \multicolumn{2}{|c|}{$\begin{array}{c}\text { Estadísticos de Colinealidad } \\
\text { VIF }\end{array}$} \\
\hline ATEN-PER & 1,068 \\
\hline CAMINO & 1,598 \\
\hline ENTORNO & 1,612 \\
\hline HORARIOS & 1,633 \\
\hline LIMP-ENT & 1,077 \\
\hline LIMP-INS & 1,669 \\
\hline LIMP-SIT & 1,124 \\
\hline NORMAS & 1,021 \\
\hline OPINPUB & 1,032 \\
\hline OPSERSUF & 1,000 \\
\hline PAISAJE & 1,194 \\
\hline RECOMPN & 1,467 \\
\hline SATGENT2 & 1,260 \\
\hline VAL-EXPEC & 1,000 \\
\hline VALORGLO & 1,615 \\
\hline VALSERAC & 1,010 \\
\hline VALVISIT & 1,000 \\
\hline VOLVER & 1,000 \\
\hline
\end{tabular}

Fuente: Elaboración propia.

Sobre el constructo SEM de partida, que sigue el esquema del citado modelo ECSI, tal como se ha referenciado en la Figura 3, después de una serie de aplicaciones y cálculos con modelos previos distintos y sus evaluaciones correspondientes, realizados con el programa SmartPLS versión 3.2.7 (Ringle et alt., 2015), se ha llegado a su reestructuración para llegar al modelo final de cálculo, tal como aparece en la Figura 4, con las variables latentes y medibles especificadas en la misma, cuya evaluación global resulta más que aceptable. En dicha Figura 4, se muestran los resultados con los coeficientes path del constructo interno y los parámetros del modelo externo o relaciones de las variables medibles con las variables latentes respectivas. Se muestran también los estadísticos de colinealidad (VIF $\geq 1$ ). En el cuadro 6 se presentan los parámetros favorables de evaluación de la fiabilidad y validez del constructo, que aseguran su aceptabilidad.

En una primera observación respecto el análisis multivariante anterior al PLS-SEM, se anota que el Factor 2 del Análisis de Componentes Principales recoge tres variables que son consideradas como el foco de resultado total o factor criterio: VALORGLO, RECOMPN y VOLVER. Precisamente las dos primeras (a las que se ha añadido como componente de satisfacción SATGENT2), se han utilizado para medir una de las variables latentes principales, dependiente en este análisis PLS-SEM: la Satisfacción Global Visitante. La variable medible VOLVER, se ha usado para medir la otra variable latente principal dependiente: la Fidelidad.

En el análisis de los resultados, se remarca la importancia del Valor Percibido, a través de las Expectativas del usuario del parque, como constructor de la Satisfacción Global del Visitante, donde tienen menor influencia la Imagen y la Calidad Percibida. 


\section{Cuadro 6 \\ EVALUACIÓN DE LA FIABILIDAD Y VALIDEZ GLOBAL DEL CONS- TRUCTO PLS-SEM}

\begin{tabular}{|c|c|c|c|c|}
\hline & Alfa de Cronbach & rho_A & Fiabilidad compuesta & Varianza extraída media (AVE) \\
\hline Fidelidad & 1,000 & 1,000 & 1,000 & 1,000 \\
\hline Satisfacción Global Visitante & 0,705 & 0,732 & 0,831 & 0,622 \\
\hline Calidad Percibida & & 1,000 & & \\
\hline Expectativas Visitante & & 1,000 & & \\
\hline Imagen & & 1,000 & & \\
\hline Quejas & & 1,000 & & \\
\hline Valor Percibido & & 1,000 & & \\
\hline
\end{tabular}

Fuente: Elaboración propia.

Respecto dicha Imagen, aportan mayores pesos la Opinión sobre las publicaciones y la Valoración de los servicios y actividades del parque. Respecto la Calidad Percibida, las variables medibles que aportan mayor peso son la Valoración del Paisaje y la Valoración del Entorno.

Sin embargo, se observa en los resultados que dicha Imagen influye poco tanto sobre la Satisfacción Global del Visitante, como sobre la Fidelización, y así mismo aporta poca influencia a la creación de expectativas del usuario o visitante del parque.

Se debe enfatizar la confirmación por el análisis PLS-SEM de que la Satisfacción Global del Visitante se refleja con un alto nivel de correlación, desde el Valor de Satisfacción Total declarada por el visitante atendiendo todas las componentes o aspectos del parque, la satisfacción derivada del ambiente y encuentro de gente, y la actitud de recomendación del parque a otras personas. Así mismo se observa un camino fuerte desde esta Satisfacción Global del Visitante hacia la Fidelización.

\section{CONCLUSIONES}

En primer lugar, y si se quiere concluir con un perfil sintético caracterizador del Visitante del Parque Nacional de Aigüestortes, se pueden anotar las siguientes puntualizaciones:

- En relación con el agrupamiento de personas, la mayoría de los visitantes encuestados iban en pareja $(55,75 \%)$, otro colectivo formaba parte de grupos $(18,36 \%)$, el $13,50 \%$ eran miembros familiares, es decir parejas con hijos, y el resto $(11,06 \%)$ eran visitantes solitarios.

- En lo que concierne a la edad de las personas encuestadas, una mayoría $(56,86 \%)$ tenían entre 26 y 45 años, y el 35,52\% restante su edad era entre 46 y 65 años.

- Respecto la residencia habitual de los visitantes encuestados estaba mayoritariamente en Cataluña en cifras de casi un 70\% (68,58\%), y el 25,66\% procedían del resto de España, mientras que sobre un $5 \%$ era la proporción de visitantes extranjeros. Los 
visitantes residentes en Catalunya eran en su gran mayoría de la provincia de Barcelona $(73,87 \%)$, a continuación, seguía Lleida con un 13,23\%, después Tarragona con un $9,35 \%$, y en última posición estaban los visitantes procedentes de Girona, con una proporción de tan solo un 3,55\%.

- En relación con el nivel de desplazamientos a la zona y el alojamiento, un $94,47 \%$ se desplazaron a la comarca con vehículo particular. Para llegar al Parque Nacional, la mayoría de los visitantes utilizaron los taxis públicos típicos en el mismo (un 68,81\%), otros colectivos accedieron a pie (un $23,01 \%$ ), un $7,52 \%$ en vehículo particular propio (situación excepcional), y el resto (solo un $0,63 \%$ ) accedieron en bicicleta.

- Sobre el total de tiempo que dedicaron a la visita del Parque Nacional, fue mayoritariamente un día $(58,19 \%)$, los visitantes que dedicaron 2 días representaron un 24,34\%, y un $17,26 \%$ de los visitantes realizó su visita tres o más días. La dedicación de menos de un día a la visita del parque fue testimonial (solamente un 0,21\% de los visitantes).

- Respecto el conocimiento del parque y preguntarles el motivo del por qué conocían el mismo, una gran mayoría de los visitantes encuestados $(64,08 \%)$ indicaron que lo conocían desde siempre, un 21,29\% que se lo habían indicado o recomendado amigos o conocidos, un 5,76\% les informaron en el hotel o en los puntos de información, un $4,21 \%$ conocieron y se motivaron por internet y un $3,55 \%$ por revistas de montaña. Es interesante remarcar que el $96 \%$ de las personas encuestadas indicó que ya conocía otros Parques Nacionales, mencionando en muchas ocasiones el Parque Nacional de Ordesa. Sin embargo, solo un 66,37 \% de los visitantes encuestados exteriorizó que conocía en mayor o menor grado la existencia de una Red de Parques Nacionales. Por otro lado, un $56.64 \%$ indicó que sabía que los Parques Nacionales tenían unos objetivos establecidos y que existían unas normas que era necesario cumplir cuando se estaba en el interior de estos, lo cual refleja que una mayoría de los encuestados tiene presente los objetivos y regulaciones de un Parque Nacional.

- En lo que concierne a las actividades a realizar dentro del parque, una gran mayoría cercana a las $3 / 4$ partes de los visitantes encuestados $(72,57 \%)$ habían planificado en mayor o menor grado la visita, y el $27,43 \%$ restante no tenían ninguna actividad planificada. Se diferenciaron las actividades a realizar entre trascendentales y consumistas (siguiendo la nomenclatura de cuestionarios utilizados anteriormente en el parque) a través de unas preguntas de respuesta múltiple y combinadas. Los que opinaron sobre actividades trascendentales, en un 50,28\% querían realizar actividades vinculadas con el senderismo y un $48,88 \%$ con el montañismo. Los que opinaron sobre actividades consumistas, indicaron que en un 45,47\% querían pasear, un 43,76\% contemplar el paisaje, un 6,67\% observar la flora y la fauna, y un 3,76\% quería hacer fotografías.

- En relación con las posibles publicaciones que habían consultado, el 73,98\% de los visitantes encuestados había consultado el folleto informativo gratuito que se ofrece en la entrada del parque o en los puntos de información, un 8,92\% de los visitantes había consultado a través de Internet, un 5,58\% en Guías de Viajes, un 4,83\% en libros editados fuera del Parque y un $3,53 \%$ en libros editados por el Parque.

- Sobre la opinión y disposición del visitante del parque a pagar una cantidad monetaria para su conservación, es decir, de si la visita requería una contraprestación monetaria, en aras a su utilización para mantenimiento de este, se evaluó mediante la pregunta 
PAGARIA. Este tipo de pregunta, que se utilizaba desde hacía un tiempo en la aplicación del método de la valoración contingente de los Espacios Naturales Protegidos (método indirecto de valoración en base del valor que le asignaban sus usuarios), al declarar su disposición a pagar una cantidad de dinero por esta visita, pensando en el mantenimiento y preservación del parque, y del coste que les suponía la visita en función de distintos factores, entre ellos el acceso. El resultado de respuesta a esta pregunta en una escala de Likert de 1 a 5 ( 1 = disposición nula a pagar, hasta $5=$ máxima disposición a pagar) demuestra una disposición neutral, ya que la media de respuestas es de 3,0487 ( 3 = disposición intermedia a pagar y por lo tanto define una posición indiferente entre disposición a pagar o no pagar). Sin embargo, se debe matizar una mayor dispersión en las respuestas que en los resultados obtenidos en otras preguntas, alcanzando una Desviación Estándar de 1,2471, frente a la media de Desviaciones Estándar del conjunto de 16 variables utilizadas en el análisis multivariante, que es de 0,805 .

La residencia habitual de los visitantes (70\% en Cataluña), nos lleva a proponer o recomendar el intento de comunicar y promocionar mejor la oferta del Parque de Aigüestortes, para tratar de aumentar la proporción de residentes del resto de España y/o venidos de otros países. Está claro que hoy día el papel de las TIC y los portales atractivos y sugerentes deben ser, entre otras, las principales herramientas de promoción-comunicación, con el apoyo también por un lado de los operadores turísticos, las Administraciones Públicas Estatal, Autonómica y Local, pensando en la actualidad sobre el papel que estan realizando las Diputaciones Provinciales.

A través de las encuestas del Parque Nacional de Aigüestortes y del Análisis de Componentes Principales, se plantea que las variables que se pueden considerar como resultado o producto de la visita serían: el Nivel de Satisfacción Total de la visita en el Parque Nacional (variable VALORGLO), la Recomendación de este Parque Nacional a otras personas (variable RECOMPN), y Si pudiera volvería a Visitar el Parque de nuevo (variable VOLVER), que en el Análisis de Componentes Principales han salido agrupadas como Factor 2.

Siguiendo con este ACP, aparte de detectar dicho Factor 2 que explica el 14,99\% de la varianza, se genera el Factor 1 que explica el 20,47\% de la varianza y determina una agrupación de las variables: LIMP-ENT, SEÑAL, CAMINO, PAISAJE y ENTORNO, que suponen valorar la percepción de elementos de calidad del Parque. Entre estos dos factores ya se explica el 35,46\% (más de 1/3) de la varianza. En orden sigue el Factor 3, que explica el 11,52\% de la varianza y acumula el 47,66\% de explicación de esta con los dos anteriores (casi la mitad), está asociado con las variables: OBJETIVO, SATGENT2 y VAL-VISI, donde las relaciones, que son positivas, indican una valoración concreta de la visita en función de los objetivos definidos, encontrar gente y por el valor de los sitios visitados. Finalmente, se han detectado dos factores más, el 4 y el 5, ambos con poco peso de explicación de la varianza $(7,01 \%$ y $6,55 \%$, respectivamente, y acumulando los anteriores se llega a un $61,22 \%$ de explicación). Analizando el factor 4 que recoge las variables: OPIN PUB y PAGARIA, se muestra una correlación negativa entre ambas, lo que plantearía un resultado que parece ilógico, que los visitantes con más disposi- 
ción a pagar mostrarían una opinión negativa o posición crítica sobre las publicaciones sobre el parque. En el análisis del factor 5, que está agrupando las variables: NORMAS, LIMP-SIT y VALSERAC, aparece una correlación negativa con esta última, que define la valoración que el visitante da a los servicios y actividades del Parque, lo que indicaría un cierto antagonismo en las opiniones que representan dicha valoración en función del conocimiento de las normas y objetivos del espacio y que podría indicar que ya conocen lo que pueden encontrarse.

Con el análisis CHAID, en la primera variable criterio Nivel de Satisfacción Total de la visita (VALORGLO), influyen la valoración del paisaje, la valoración de la visita, la señalización y la consecución de los objetivos iniciales, pero no afectan el resto de las variables. En la segunda variable criterio Recomendaría usted el Parque Nacional (RECOMPN), se detecta que guarda relación con la posibilidad de pagar, la valoración de la visita, la consecución de los objetivos iniciales y la satisfacción por la cantidad de gente encontrada, y no afectan el resto de las variables. Con lo cual, las variables sobre el conocimiento de las normas, la limpieza de los sitios visitados y del entorno, la valoración del entorno, la valoración de los servicios y las actividades y la opinión de las publicaciones, no se refleja que han influido en los resultados de las dos variables criterio.

El análisis CHAID nos ha llevado a unas conclusiones partiendo de la base que respecto el Nivel de Satisfacción Total de la visita (VALORGLO, con escala de Likert $1,2,3,4$ y 5), nadie declaró muy poca satisfacción ( $0 \%$ escala 1$)$, tan solo 5 visitantes declararon poca satisfacción (1,11\% escala 2), 45 visitantes alguna satisfacción (9,29\% escala 3), 181 visitantes bastante satisfacción (40,04\% escala 4) y 224 visitantes mucha satisfacción (49,56\% escala 5). Es decir, se concluye que cerca del 90\% (exactamente un $89,60 \%$ ) de los visitantes declararon que estaban bastante o muy satisfechos. A través del dendrograma, se visualiza en una primera partición la influencia del valor del paisaje (PAISAJE) que engloba 2 grupos significativos (grupos 1 y 2): el primero de 448 visitantes que recoge las escalas de dicha variable 1,2, 4 y 5 , y el segundo grupo que recoge solo a 4 visitantes que declararon la escala 3. Este primer grupo de 448 visitantes, en un siguiente nivel queda partido en tres nuevos grupos (grupos 3, 4 y 5) integrando a la variable PAISAJE, la variable VAL-VISI o Valoración del nivel de satisfacción sobre los sitios visitados y su valor socioeconómico; en el grupo 3 se recogen 222 visitantes que aparte de declarar las escalas anteriores 1, 2, 4 y 5 de PAISAJE, declaran las escalas 1 y 4 de VAL-VISI; en el grupo 4, 77 visitantes declaran las escalas 2 y 3 de VAL-VISI y en el grupo 5, 149 visitantes declaran el máximo valor 5 de satisfacción en VAL-VISI. En un cuarto nivel, el grupo 3 de 222 visitantes da lugar a una bipartición: grupo 6 de 163 visitantes donde se añade la variable SEÑAL o Valoración de la señalización de los caminos que valoran en las escalas 1, 2, 3 y 5 , y en el grupo 7 de 59 visitantes, valoran señal según escala 4; siguiendo el cuarto nivel, el grupo 5 de 149 visitantes se parte en el grupo 8 con 145 visitantes (casi todos los del grupo 5) que han declarado las escalas de SEÑAL $1,3,4$ y 5 , y en el grupo 9 que solo contiene 4 visitantes que han declarado la escala 4 de señal. Finalmente, en un último quinto nivel, el grupo 8 se particiona en el grupo 10 con 73 visitantes (más o menos la mitad) que han señalado en adición la variable OBJETIVO (Valoración del objetivo conseguido sobre la realización de la actividad prevista) en sus escalas 1, 2, 3 y 5, y en el grupo 11 con 72 visitantes (la otra mitad) con la escala 4 de la 
variable OBJETIVO. Concluir que, respecto el Nivel de Satisfacción Total de la visita, el análisis CHAID ha aglutinado a los visitantes en varios niveles en distintos grupos según las variables PAISAJE, VAL-VISI, SEÑAL y OBJETIVO, que parece que tienen amplia resonancia y se consideran fundamentales en relación con el citado Nivel de Satisfacción Total de la visita.

Respecto la variable RECOMPN (Recomendaría este Parque Nacional a otras personas), el análisis CHAID nos ha facilitado también otras conclusiones. Esta variable daba opción a declarar cinco niveles distintos de recomendación, desde 1 = nula o muy poca recomendación, 2 = poca recomendación, 3 = recomendación media, 4 = alta recomendación, y 5 = muy alta recomendación. De los 452 visitantes encuestados, 54 visitantes declararon recomendación media $(11,95 \%), 228$ visitantes señalaron el nivel de alta recomendación $(50,46 \%)$ y 170 visitantes expresaron en la encuesta muy alta recomendación $(37,61 \%)$. Es decir, se concluye que un $88,07 \%$ decidieron señalar los dos niveles más altos de recomendación. Del segundo dendrograma que corresponde a esta variable RECOMPN, se presenta un primer nivel de tripartición de los 452 visitantes agregando la variable VAL-VISI en un grupo 1 de tan solo 22 visitantes que respondieron con los niveles de escala 1 y 2 (respectivamente, muy poco y poco nivel de satisfacción sobre los sitios visitados y su valor socioeconómico) de VAL-VISI, un grupo de 282 visitantes que contestaron la escala 3 y 4 (algún nivel de satisfacción y bastante nivel de satisfacción) de VAL-VISI y el grupo 3 de 149 visitantes que declararon la escala máxima 5 (mucho nivel de satisfacción). En el ulterior nivel de particiones, el grupo 2 anterior de 282 visitantes se particiona en dos grupos agregando la variable CAMINO (Estado de los caminos): en el grupo 4 de 255 visitantes con respuesta en las escalas 1, 2, 4, y 5, y en el grupo 5 de 27 visitantes con respuesta en la escala 3 de dicha última variable. En este mismo nivel particional el grupo 3 de 149 visitantes se divide en dos grupos también que agregan la variable PAGARIA (Le parecería adecuado pagar para poder mantener el Parque): el grupo 6 de 92 visitantes que declararon en PAGARIA las escalas 1, 4 y 5, y el grupo 7 de 57 visitantes que expresaron en su encuesta las escalas 2 y 3 . Finalmente, en el último nivel de particiones, el grupo 6 anterior (con 92 visitantes) se divide en dos grupos integrando la variable OBJETIVO (Valoración del objetivo conseguido sobre la realización de la actividad prevista): el grupo 8 con tal solo 2 visitantes que declaran las escalas menores 1 y 2 , y el grupo 9 de los 90 visitantes restantes que declaran las escalas de mayor valor 3, 4 y 5; por otro lado en este mismo nivel particional, el grupo 7 (con 57 visitantes) también se divide en dos grupos agregando la variable SATGENT2 (Satisfacción en función de la gente encontrada): el grupo 10 de 49 visitantes que declararon las escalas 1, 2, 3 y 5 de SATGENT2, y el grupo 11 de 8 visitantes que declararon la escala 5 de esta última variable. Concluir respecto esta otra variable criterio RECOMPN, que el análisis CHAID ha combinado y aglutinado las variables VAL-VISI, CAMINO, PAGARIA, OBJETIVO y SATGENT2, lo que implica la importancia del valor del nivel de satisfacción sobre los sitios visitados y su valor socioeconómico, el estado de los caminos, la disposición a pagar por el mantenimiento del parque, el que se haya valorado el objetivo conseguido sobre la realización de la actividad prevista y la satisfacción al haber encontrado gente. Todo ello influye en el nivel de recomendación de la visita del parque a otras personas. 
Con el análisis PLS-SEM se puede concluir el grado de importancia del Valor Percibido, a través de las Expectativas del usuario del parque, como constructor de la Satisfacción Global del Visitante, donde tienen menor influencia la Imagen y la Calidad Percibida. En relación con la Imagen, aportan mayores pesos la Opinión sobre las publicaciones y la Valoración de los servicios y actividades del parque. Respecto la Calidad Percibida, las variables medibles que aportan mayor peso son la Valoración del Paisaje y la Valoración del Entorno. Remarcar también como conclusión, la confirmación a través del análisis PLS-SEM, que la Satisfacción Global del Visitante se manifiesta con un alto nivel de correlación, desde el Valor de Satisfacción Total declarada por el visitante atendiendo todas las componentes o aspectos del parque, la satisfacción derivada del ambiente y encuentro de gente, y la declaración de recomendación del parque a otras personas. Así mismo se observa un camino fuerte desde esta Satisfacción Global del Visitante hacia la Fidelización.

Sería importante estudiar la forma de identificar mejor y consolidar los ítems/variables que podrían reflejar la sensación de satisfacción y de cumplimiento de las expectativas de la visita al parque y en general a un Espacio Natural Protegido y su relación con la calidad del espacio, pues a través de las actuales encuestas a veces pueden surgir algunas dudas sobre su idoneidad. El hecho de que exista relación entre el conocimiento y la información previa del Parque Nacional y el nivel de satisfacción final, induce a concluir que no afecta el factor sorpresa y ya se conocen los servicios, prestaciones y características del entorno, por parte de una mayoría de los visitantes.

A través de los esfuerzos y actuaciones de Europarc-España y de las Administraciones Públicas, la Red de Parques Nacionales y también globalmente todos los Espacios Naturales Protegidos, gozan del soporte idóneo para poder conjugar la conservación natural y la protección de la biodiversidad, con el uso ecoturístico de los mismos y su disfrute, a través de Planes Rectores de Uso y Gestión (PRUG), que se deben acomodar para la reunión de todos los objetivos ecológicos y de uso y disfrute. Será muy importante, el papel académico de seguimiento con programas de investigación, que vayan secundando y mejorando dichos objetivos, entre ellos la satisfacción de los visitantes ecoturísticos.

\section{REFERENCIAS BIBLIOGRÁFICAS}

BLANCO, R. y BENAYAS, J. (1994): "El turismo como motor de desarrollo rural. Análisis de los proyectos de turismo subvencionados por Leader I". Revista de Estudios Agro-Sociales, $\mathrm{n}^{\mathrm{o}}$ 169, pp 119-147.

DÍAZ-PEREZ, F. y BETHENCOURT-CEJAS, M. (2016): “CHAID algorithm as an appropriate analytical method for tourism market segmentation". Journal of Destination Marketing \& Management, vol. 5 (3), pp. 275-282.

DONAIRE, J.A. (2002): "Ecoturisme en zones de muntanya", en IV Congrés Internacional del Medi Ambient d'Andorra. Andorra, Centre de Biodiversitat. Institut d'Estudis Andorrans, 8 p.

EUROPARC-ESPAÑA. (2005): Manual sobre conceptos de uso público en los espacios naturales protegidos. Madrid, Fundación Fernando González Bernáldez, Serie manuales EUROPARC-España. 
FLORENSA GUIU, R. (2015): Evaluación de la calidad y satisfacción del usuario de los Espacios Naturales Protegidos de Montaña. Aplicación al Parc Nacional d'Aigüestortes $i$ Estany de Sant Maurici. Tesis doctoral. Departamento de Administración de Empresas. Universidad de Lleida.

FORNELL, C. (1992): “A National Customer Satisfaction Barometer: The Swedish experience", Journal of Marketing, vol. 56 (1), pp. 6-21.

GARCÍA LÓPEZ DE MENESES, T. y BARRENA FIGUEROA, R. (2013): "Preferencias del visitante de establecimientos de turismo rural. Estudio en Navarra". Cuadernos de Turismo, no 32 , pp. 141-153.

GÓMEZ-LIMÓN, J. GUZMÁN, A. y DE ANDRÉS, A. (2010): Guía para la adhesión de las empresas turísticas a la Carta Europea de Turismo Sostenible en espacios protegidos. Madrid, EUROPARC-España. 2010, y Fundación Fernando González Bernáldez.

HARMAN, H. (1976): Modern Factor Analysis. Chicago, The University of Chicago Press.

KASS, G.V. (1980): “An exploratory technique for investigating large quantities of categorical data”. Applied Statistics, vol. 29 (2), pp. 119-127.

KIM, S.S, TIMOTHY, D.J. y HWANG, J. (2011): “Understanding Japanese tourists' shopping preferences using the decision tree analysis method". Tourism Management, vol. 32 (3), pp. 544-554.

MAGIDSON, J. (1994): “The CHAID Approach to Segmentation Modeling: CHI-squared Automatic Interaction Detection", en Richard P. Bagozzi (Ed.), Advanced Methods of Marketing Research, Basil Blackwell, Ltd., Cambridge, Massachusetts (USA), pp. 118-159.

PÉREZ DE LAS HERAS, M. (2004): Manual del turismo sostenible. Madrid, Mundi Prensa.

PICÓN PRADO, E., VARELA MALLOU, J. y LÉVY MANGIN J.P. (2004): Segmentación de mercados: aspectos estratégicos y metodológicos. Madrid, Financial Times/ Prentice Hall.

RINGLE, CH. M. WENDE, S. y BECKER, J.M. (2015): SmartPLS 3. Publisher: SmartPLS GMBH. Disponible en "http://www.smartpls.com”, version 3.2.7, año 2015.

RUMMEL, R.J. (1970): Applied Factor Analysis. Evanston, Northwestern University Press.

SANTESMASES MESTRE, M. (2009): Diseño y Análisis de Encuestas en investigación social y de mercados (DYANE v4). Madrid, Ediciones Pirámide.

SECRETARÍA GENERAL DE TURISMO (SGT) (2004): Estudio sobre el turismo de naturaleza en España y su plan de impulso. Madrid, Ministerio de Industria, Turismo y Comercio.

STEWARD, D.W. (1981): "The Application and Misapplication of Factor Analysis in Marketing Research". Journal of Marketing Research, vol. XVIII (1), pp. 51-62.

TENENHAUS, M., VINZI, V.E., CHATELIN, Y.M, y LAURO C. (2005): "PLS path modeling”. Computational Statistics and Data Analysis, vol. 48 (1) , pp. 159-205. 
